\section{Revue de Sémantique et Pragmatique}

$37 \mid 2015$

Sémantique et pragmatique

\title{
La France de la débrouille : étude ethnoaxiologique d'une valeur culturelle hypothétique
}

\section{Bert Peeters}

\section{(2) OpenEdition}

\section{Journals}

Édition électronique

URL : http://journals.openedition.org/rsp/1242

DOI : $10.4000 /$ rsp. 1242

ISSN : 2610-4377

Éditeur

Presses universitaires d'Orléans

\section{Édition imprimée}

Date de publication : 1 juin 2015

Pagination : 103-122

ISSN : 1285-4093

\section{Référence électronique}

Bert Peeters, «La France de la débrouille : étude ethnoaxiologique d'une valeur culturelle

hypothétique ", Revue de Sémantique et Pragmatique [En ligne], 37 | 2015, mis en ligne le 01 juin 2016, consulté le 03 juillet 2020. URL : http://journals.openedition.org/rsp/1242 ; DOI : https://doi.org/

$10.4000 /$ rsp. 1242 


\section{LA France de LA dÉBROUILLE : ÉTUDE ETHNOAXIOLOGIQUE D'UNE VALEUR CULTURELLE HYPOTHÉTIQUE}

Bert Peeters

Griffith University, Brisbane, b.peeters@griffith.edu.au

Dans le cadre méthodologique de l'ethnolinguistique appliquée, élaborée par l'auteur de ces lignes à des fins didactiques (voir ci-dessous), l'ethnoaxiologie est la démarche qui consiste à corroborer la réalité, dans une langue-culture donnée, de valeurs culturelles soit purement hypothétiques, soit d'ores et déjà plus ou moins communément associées à la langue-culture concernée, en repérant dans celle-ci des indices saillants, linguistiques aussi bien que non linguistiques, qui en constituent un reflet fiable. L'objectif de l'ethnolinguistique appliquée dans son ensemble est de proposer des pistes de recherche exploitables dans les cours avancés de FLE (mais aussi d'autres langues étrangères); il s'agit de permettre à des étudiants ayant acquis des bases linguistiques relativement solides de partir à la découverte des valeurs culturelles d'une langue-culture à travers la langue qu'ils étudient (cf. Peeters 2013a).

Plutôt que de rendre compte de l'exacte procédure à suivre et de multiplier les conseils pratiques et les renvois aux dispositifs à mettre en place en vue de faciliter la démarche ethnoaxiologique, nous rapportons dans ce qui suit les résultats d'une recherche dont le but était de corroborer la réalité de la valeur culturelle présumée de la débrouille, à partir d'indices non envisagés lors de la formulation de l'hypothèse (qui, elle, reposait sur une étude ethnophraséologique des tournures On va s'arranger et On s'arrangera; Peeters 2006a, 2014). Nous commencerons par représenter la valeur culturelle hypothétique sous forme d'un scénario culturel $(\S 1)$. Il sera ensuite montré que la débrouille occupe effectivement une place importante au sein de la langue-culture française et qu'elle y est valorisée ( $§ 2$ ). La corroboration linguistique qui suit en apportera 
des preuves supplémentaires $(\S 3)$. Aucun doute ne devrait plus être permis au bout de ce double examen : la débrouille a effectivement toutes les chances d'être une valeur culturelle française, et à ce titre mérite d'être portée à l'attention de tout apprenant avancé du FLE (français langue étrangère) (§ 4). ${ }^{1}$

\section{LA DÉBROUILLE : UN SCÉNARIO CULTUREL}

Le but des scénarios culturels proposés au sein de l'ethnolinguistique appliquée, et de manière plus générale dans l'ensemble des travaux inspirés par l'approche MSN,${ }^{2}$ est d'expliciter, en termes clairs, précis et universellement accessibles, les valeurs culturelles et autres façons de penser qui ont cours dans une langue-culture particulière, les intuitions d'ordre évaluatif qui existent au sein de cette langue-culture et qui portent sur ce qui y est bien et mal vu, sur ce qu'il convient et ce qu'il ne convient pas de dire, de penser, de sentir ou de faire. Le scénario culturel correspondant à la valeur hypothétique de la débrouille, dont le présent exposé se propose de corroborer la réalité, est le suivant :

Scénario culturel explicitant la valeur culturelle française de la débrouille

(a) c'est bien que les gens pensent comme ça :

(b) parfois, certaines choses ne sont pas comme je veux qu'elles soient

(c) si c'est comme ça, c'est bien de faire quelque chose

(d) si je peux faire quelque chose, ce n'est pas bien que je ne fasse rien

(e) à cause de cela, si je peux faire quelque chose, je le fais

(f) quand je fais cette chose, je sens quelque chose de bien

(g) après que j'ai fait cette chose, je sens quelque chose de bien

(h) beaucoup de gens ici pensent comme ça

(i) ils savent que quelque chose de mal peut leur arriver s'ils ne pensent pas comme ça

1 Nous remercions les relecteurs de leurs remarques critiques, qui nous ont permis de préciser un certain nombre de points. Les imperfections qui restent ne sont imputables qu'à nous.

2 Traduction de l'anglais NSM approach, où NSM = natural semantic metalanguage. La MSN ou métalangue sémantique naturelle, associée aux publications d'Anna Wierzbicka et de Cliff Goddard, est un outil descriptif d'une clarté maximale, culturellement neutre (autant que cela faire se peut), et dont la rigueur n'est plus à démontrer. Elle permet, grâce à son lexique et à sa grammaire empiriquement validés comme étant universels (c'est-à-dire transposables dans toutes les langues du monde sans aucune déformation sémantique), de rendre compte de tout ce qui, dans une langue-culture, est culturellement spécifique : d'une part, le sens des mots, des tournures courantes, des expressions idiomatiques, des structures syntaxiques, etc., et de l'autre, les comportements communicatifs, les normes communicatives et les valeurs culturelles des locuteurs. Pour de plus amples informations en langue française, on verra Wierzbicka (2006a, b) et Peeters (2010, 2012). Les férus de détails techniques trouveront de quoi se satisfaire dans Goddard \& Wierzbicka (2002) et, pour les langues romanes, Peeters (2006b). Pour une introduction moins technique, voir Goddard (2011). 
Il n'y a pas de consensus quant à la meilleure façon de présenter les scénarios culturels qui ont cours dans une langue-culture particulière. Le gabarit adopté ici constitue une nouvelle tentative de rendre compte, de manière intuitivement correcte, de façons de penser classées comme des valeurs culturelles. Les composantes (a) et (h)-(i) servent de cadre au scénario, soulignant qu' on a affaire à une façon de penser valorisée et relativement répandue dans la langue-culture française. La façon de penser elle-même est explicitée dans les composantes (b) à (g), qui constituent le « corps » du gabarit. Il faut d'abord oser admettre que tout ne va pas toujours pour le mieux (composante b). Il importe alors d'essayer de trouver un remède (composantes $\mathrm{c}$ à e), dont la mise en place est source de satisfaction (composantes $f$ et $g$ ).

\section{SAILLANCE CULTURELLE ET VALORISATION DE LA DÉBROUILLE DANS LA LANGUE-CULTURE FRANÇAISE}

"Spécificité française ${ }^{3},{ }^{3}$ "notion typiquement française $~^{4}$ : voilà comment les Français eux-mêmes s'expriment au sujet de leur débrouille - qu'ils prennent décidément très à cœur. Quand un comportement particulier, par exemple la débrouille, fait l'objet de témoignages explicites et qu'il se manifeste dans des faits de société, on peut légitimement conclure qu'il est culturellement saillant. Pour arriver à la conclusion qu'il est valorisé, il faut aller plus loin et procéder à une double interrogation. Comment les faits de société sont-ils perçus au sein de la langue-culture concernée ? Est-ce que les observateurs se contentent d'observer ou bien prennent-ils position ? On verra à la lecture de ce qui suit que, dans la langue-culture française, la débrouille est effectivement valorisée.

\subsection{L'ÉCART ENTRE RÈGLES ET PRATIQUE}

Dans la vie publique, et en particulier au sein des entreprises, la débrouille se manifeste en premier lieu dans ce que d'Iribarne (2006, 129) appelle «l'écart massif » entre « ce que l'on est censé faire et ce que l'on fait habituellement », entre « les principes solennellement affichés et les arrangements qui y dérogent», entre les règles et la pratique. Il s'agit, toujours selon d'Iribarne, d'un trait bien connu de la société française, où on parle de grève du zèle quand les règlements sont respectés à la lettre - «et l'étendue des troubles qui en résultent montre à quel point ces règlements n'ont pas été conçus pour être appliqués » (ibid.). Aux yeux de la plupart des Français, l'écart entre règles et pratiques n'a le plus souvent rien de " honteux » (ibid., 131) : "C'est, au contraire, celui qui prétendrait prendre les règles tout à fait au sérieux qui se ferait mal voir,

3 Propos du sociologue Michel Crozier recueillis par Pascale-Marie Deschamps, Enjeux Les Échos, 01/03/05.

4 Asselin \& Mastron $(2005,93)$. 
apparaissant selon les cas comme une sorte de dangereux ayatollah ou comme un lourdaud ridicule, ignorant des finesses du monde ». L'écart entre règles et pratiques est en fait si répandu et si normal qu'à en croire d'Iribarne (ibid., 130), « ne pas le rencontrer ailleurs [c'est-à-dire à l'étranger; B.P.] suscite une sorte de choc culturel ». Le sociologue français cite Bourrier $(1999,193)$, aux yeux de qui la conformité aux règles dans la centrale nucléaire américaine dont elle a étudié le fonctionnement constitue un "phénomène assez inattendu surtout au regard de l'expérience française ».

\subsection{DÉBROUILLE ET DEMANDE D'ASSISTANCE OU DE FAVEURS}

En France, les tentatives d'obtenir quelque chose rien qu'en y mettant du sien, c'est-à-dire sans se faire assister, sont souvent perçues comme étant vouées à l'échec. Se vanter auprès de quelqu'un d'avoir réussi dans la vie sans « se faire pistonner » (cf. § 3.1.7) suscite l'incrédulité. L'Américaine Elizabeth Bard $(2010,311)$ rapporte l'expérience suivante arrivée à son mari français Gwendal au cours d'un entretien d'embauche en France :

The idea that he [le mari ; B.P.] busted his ass, flew back and forth to LA, spoke English, wrote letters, took meetings, crunched numbers - none of this made the slightest bit of sense to the people in front of him. The cinema industry, like many professions in France, is filled with fils de, literally « sons of », people who got where they are with family connections and family money. (...)

Finally Gwendal just gave up and did it the French way. "You know, my wife is American. She's actually Jack Warner's granddaughter. » (I'm not.)

«Ahhh, bien oui.» There was a huge exhalation and nods of comprehension around the table.

They simply couldn't conceive of the fact that he'd gotten there any other way.

Au lieu de tout faire selon les règles, de façon « correcte », mieux vaut trouver quelqu' un à qui on peut demander une « petite faveur», éventuellement moyennant un «petit cadeau » ou une autre forme de récompense, et qui peut faire jouer son influence. Au lieu de perdre du temps en essayant d'obtenir ce qu'on se verra probablement refuser, mieux vaut faire des démarches auprès de fonctionnaires que l'on connait personnellement ou à qui on a accès grâce à des amis communs, et à qui on demande d'intervenir parce que les contraventions pour parking illégal se sont accumulées pendant un séjour à l'étranger (Loomis 2001, 77), qu'un visa est sur le point d'expirer (Baxter 2006, 122-123), que d'autres lettres ou pétitions n'avaient pas apporté de solution (Lewis 2006, 246), etc. Le fait que de «nouveaux Français » installés en France en font état dans le récit de leurs expériences montre que la pratique est nettement plus répandue que dans leur pays d'origine et que personne ne trouve à y redire. 


\subsection{LA DÉBROUILLE DANS LA VIE DOMESTIQUE}

"The French practice la débrouillardise in all aspects of life, whether in getting a broken electrical appliance to work, in cutting in lines, or in finding a way to pay less tax ». Le témoignage de l'Australien Ross Steele $(2006,101)$ permet de passer de la vie publique (d'où il tire deux de ses exemples, griller les files d'attente et réduire le montant des impôts) à la vie domestique (d'où il tire le troisième, fixer un appareil électroménager). La débrouille chez soi fait l'objet de nombreuses émissions télévisées telles que, par exemple, La France de la débrouille (France 2, 15 janvier 2009) et Les rois de la débrouille, 3 ans après (France 2, 30 avril 2011). Le deuxième reportage fait suite au précédent : on y retrouve en partie les débrouillards du reportage plus ancien. ${ }^{5}$ Il y en a d'autres dont le titre renvoie au système $D$ (cf. § 3.1.5) : Les rois du système D (TF1, 13 avril 2007, 14 mars 2008), Bons plans, combines et système D : la France de la débrouille (M6, 6 avril 2008), La France du système D (France 5, 2 novembre 2008), Besoin d'argent : enquête sur les nouveaux rois du système $D$ (M6, 26 avril 2009). La liste continue et prouve que la débrouille est une valeur très prisée dans la langue-culture française. Il existe par ailleurs des magazines illustrés tels que Système $D$, publié depuis la fin de la Deuxième guerre mondiale, qui se décrit comme « le mensuel du bricolage » et s'adresse à ceux qui veulent « tout faire et réussir à la maison et au jardin ».

\section{CORROBORATION LINGUISTIQUE}

On aurait tort, pour prouver la saillance culturelle et la valorisation de la débrouille dans la langue-culture française, de se contenter des observations qui précèdent. Plusieurs faits de langue semblent à leur tour inviter la conclusion que la débrouille est une valeur culturelle, inscrite dans la langue de tous les jours ; ces faits de langue seront illustrés à l'aide d'extraits de presse tirés pour la plupart de la banque de données Factiva (www.factiva.com) ${ }^{6}$

5 D comme débrouille (Canal+, multiples rediffusions), en revanche, est une série dont l'animateur, Sebastian Perez Pezzani, part à la découverte d'autres pays (les États-Unis, le Rwanda, le Vietnam, le Kenya...), où la débrouille est censée être un art de vivre autant qu'en France.

${ }^{6}$ Légende $: \mathrm{BR}=$ Le Berry Républicain, $\mathrm{CL}=$ La Charente Libre, $\mathrm{CP}=$ Centre Presse, $\mathrm{E}=$ Les Échos, $\mathrm{ER}=$ L'Est Républicain, $\mathrm{Ex}=$ L'Express, $\mathrm{JF}=$ Journal des Finances, $\mathrm{L}=$ Libération, $\mathrm{M}=$ Le Monde, $\mathrm{Ma}=$ Marianne, $\mathrm{ML}=$ Midi Libre, $\mathrm{NR}=$ La Nouvelle République, $\mathrm{OF}=$ Ouest France, $\mathrm{P}=$ Le Parisien, $\mathrm{PME}=$ PME Magazine, $\mathrm{Pr}=$ Le Progrès, $\mathrm{Pt}=$ Le Point, $\mathrm{Pv}=$ La Provence, $\mathrm{SO}=$ Sud Ouest, $\mathrm{St}=$ Stratégies, $\mathrm{T}=$ La Tribune, Té $=$ Le Télégramme, $\mathrm{VN}=$ La Voix du Nord. Certains exemples ont été légèrement raccourcis. 


\subsection{MOTS D'USAGE FRÉQUENT}

Parmi les mots d'usage fréquent autres que le verbe s'arranger (Peeters 2014), certains rappellent de façon immédiate la valeur culturelle à l'étude : ce sont le verbe se débrouiller et ses dérivés débrouille et débrouillard(e). ${ }^{7}$ Il convient de mentionner en outre - sans prétendre à l'exhaustivité - le verbe se démerder et ses dérivés démerde, démerdard(e) et démerdeur, de même que les vocables système $D$, tuyau et piston, et les dérivés de ce dernier. Se débrouiller et se démerder se prêtent à la plupart des usages courants du verbe s'arranger (Peeters 2014).

\subsubsection{Se débrouiller}

Devant une difficulté, attendue ou non, on peut dire - et on dit souvent On va se débrouiller, ou encore Je vais me débrouiller. S'engager de la sorte, sans s'en cacher, prouve que la débrouille est valorisée et qu'elle est source de satisfaction (cf., dans le second exemple, l'exclamation Voilà on y arrive).

Il y a des plans B, on va se débrouiller. (Té 16/02/11)

B Vous voulez me donner de la monnaie?

C Heu... vingt centimes c'est tout ce que j'ai...

B Heu non ça va pas m'arranger merci (sourire).

C Excusez-moi.

B Oh mais c'est rien, je vais me débrouiller. Alors, sur deux cents francs ça fait cent quatre-vingt-six trente... cent, cinquante, soixante, soixante-dix, hum quatre-vingt-cinq, quatre-vingt-six... vingt et trente, voilà on y arrive.

(Échange entre client et vendeuse dans une boulangerie de Lyon, KerbratOrecchioni 2008, 105)

Le verbe se débrouiller parle à l'imagination de plusieurs auteurs de langue anglaise, qui n'hésitent pas à y recourir tout en s'exprimant dans leur propre langue. Fiona McGregor est l'auteure d'un roman australien intitulé Au pair ; Chantale y encourage son amie, à qui elle a sous-loué une chambre qu'elle ne pourra pas garder, à ne pas s'inquiéter :

I'm sorry about the room, Sophie, she said. But don't worry. Tu te débrouilleras. (McGregor 1993, 27)

Jeffrey Greene, poète américain et auteur d'un récit véridique intitulé French spirits, quant à lui, décrit les efforts culinaires de sa mère :

Though the butter cream frosting was a little loose, my mother improvised, getting enough of the butter cream to act like stucco. In spite of her chaotic methods, in the end elle se débrouille, as the French say. She manages. (Greene 2002,134)

Steele $(2006,101)$ faitremarquerque le compliment Il/Elle saitse débrouiller « is always sincere and accompanied with a twinkle in the eye ».

\footnotetext{
7 Le mot débrouillardise, par contre, est d'une fréquence nettement moindre.
} 


\subsubsection{La débrouille}

«Seitich denken kann, spricht man in Frankreich von "chômage", "précarité" und "la débrouille" (Arbeitslosigkeit, Präkariat und sich durchschlagen) », déclare André Lehmann (Sonntagszeitung, 12/08/12). "Ich komme aus einem Land, in dem das Wort "débrouille", das so viel wie "geschicktes sich aus der Affäre ziehen" bedeutet, ein Grundbegriff ist », dit pour sa part Georges Marion (Süddeutsche Zeitung, 18/01/03), s'exprimant lui aussi en allemand au sujet de la «notion fondamentale » de la débrouille dans son pays d'origine. Le nom débrouille apparait avec une certaine fréquence sous la dépendance d'un autre nom auquel il est lié à l'aide de la préposition de. À côté de la France de la débrouille, syntagme qui sert notamment de titre à cet article et à certaines émissions télévisées (cf. § 2.3), on parle d'art de la débrouille, d'économie de la débrouille, on se forme à l'école de la débrouille (qui est une forme d'autoapprentissage), on est ou on devient roi ou reine de la débrouille, etc. :

Eh oui, à l'heure de la multiplication des chaines et de l'arrivée de BeIn Sport 1, la chaine sportive d'Al Jazeera, suivre l'une des plus grandes compétitions se conjugue avec l'art de la débrouille. (OF 16/06/12)

La mode est à la consommation collaborative, entendez par là l'économie de la débrouille. La vente, la location ou le troc entre particuliers séduisent de plus en plus d'adeptes. (VN 13/08/13)

C'est le prix à payer lorsque l'on veut faire du cinéma en dehors du circuit professionnel classique. C'est l'école de la débrouille. (ML 28/04/14)

Roi de la débrouille, il tourne ses courts métrages avec très peu de moyens. (Pv 25/08/10)

Parmi les habitués, Marine, étudiante, la reine de la débrouille. Tout ce qu'elle achète ici, elle le revend plus cher sur internet. (Extrait de l'émission La France de la débrouille; $\mathrm{cf}$. § 2.3)

Dans l'usage de tous les jours, quand ils ne sont pas utilisés ironiquement, les mots art, école, roi et reine, plus sans doute que le mot économie, soulignent le côté positif de la débrouille. Au pluriel, la métaphore rois de la débrouille s'applique souvent aux Français dans leur ensemble, même s'ils ne sont pas les seuls à avoir été décrits de la sorte et que d'autres se sont vu désigner de la même façon. La métaphore se manifeste soit sous forme d'apposition, soit avec un verbe copule. Les deux exemples ci-dessous peuvent à juste titre être considérés comme des témoignages explicites de la valorisation de la débrouille (nonobstant la présence, dans le premier, d'un mécanisme de distanciation, l'incise dit-on) : ${ }^{8}$

Les Français, dit-on, sont les rois de la débrouille. (St 15/11/07)

Les Français, rois de la débrouille, achètent désormais en plus petites quantités. (TF1, journal télévisé, 24/02/09)

\footnotetext{
8 Une autre métaphore « royale », rois du système $D$, sera illustrée dans ce qui suit (§ 3.1.5).
} 
On a aussi la tournure C'est la foire à la débrouille, qui insiste sur le nombre et la variété de démarches possibles en vue de se débrouiller :

Trouver un bureau à l'assemblée, c'est un peu la foire à la débrouille. (Pr 27/06/07)

Hormis les syntagmes à préposition, il y a enfin des composés d'un type plus moderne. La génération débrouille est celle des jeunes qui savent faire preuve d'ingéniosité en temps de crise, quand le pouvoir d'achat est affecté et qu'il faut user de moyens divers pour consommer à moindre cout. Système débrouille, quant à lui, sera évoqué ci-dessous (§ 3.1.5), dans le volet consacré au « système $\mathrm{D}$ ».

\subsubsection{Débrouillard}

Dans un texte publié sur internet, cité notamment par Klein $(2013,32)$, l'économiste américain Robert Neuwirth s'exprime comme suit :

The French have a word that they often use to describe particularly effective and motivated people. They call them débrouillards. To say a man is a débrouillard is to tell people how resourceful and ingenious he is.

Une version un tant soit peu corrigée figure dans Neuwirth $(2011,17)$ : «To say a man (or woman) is a débrouillard(e) is to tell people how resourceful and ingenious he or she is ». Que les Françaises méritent mieux que d'être ignorées ou mises entre parenthèses ressort clairement de ce témoignage repéré dans la presse étrangère :

The job situation in France is even worse than North America yet I'm constantly amazed by how débrouillardes [resourceful] French women are. They have the inner strength and equilibrium to do what it takes, see their situation as a phase and rise to the occasion. They will take a bad job and see it as a temporary thing to help the family. They stay positive and sensible, making do with less in ingenious ways. ${ }^{9}$

À ces témoignages d'observateurs externes, regardant la langue-culture française de l'extérieur, on peut en ajouter d'autres, internes, reposant sur le même adjectif précédé du verbe copule être. "Les cons, c'est connu, ce sont les Belges. Le Français, lui, est débrouillard, donc le contraire d'un imbécile, O.-K. ? » L'auteur de ces propos provocateurs n'est autre que Bertrand PoirotDelpech, de l'Académie française (M 17/05/00). Après un énième weekend particulièrement meurtrier sur les routes de la République, l'illustre écrivain déclare ses concitoyens champions de la déculpabilisation, qu'il fait passer ironiquement comme un exemple (très peu flatteur) de débrouille. Accusé

9 Propos de Mireille Guiliano, auteure du bestseller French women don't get fat, cités dans The Globe and Mail, 27/10/09. Cf. aussi Stefan Brändle (Der Standard, 14/05/03) : "Wieder einmal beweisen die Französinnen und Franzosen, dass sie Meister in der "débrouille" sind, dem Sich-aus-der-Patsche-Helfen ». 
d'irresponsabilité sur les routes, le Français «se débrouille » en disant qu'il n'est ni plus ni moins coupable que ceux qui l'entourent, et du coup il a la conscience tranquille.

D'autres observateurs, moins connus que Poirot-Delpech, ont qualifié les Français de « débrouillards ». Tantôt, ils opposent ou juxtaposent des traits trouvés dans des langues-cultures distinctes :

On voulait un réseau très dynamique qui prenne le meilleur des cultures française et américaine : les Français sont créatifs et débrouillards, et la qualité de leur formation en fait des ingénieurs recherchés ; les Américains ont le gout du risque, ils sont portés vers l'action, cultivent une approche positive et savent nouer facilement un contact avec les gens. (E 23/09/02)

Le Suisse est plus travailleur, le Français plus débrouillard. (PME 30/06/05)

Tantôt ils ne regardent pas au-delà des frontières de l'Hexagone :

Les Français sont débrouillards. Quand les jeux de la Française des jeux passeront en euros, ils comprendront très vite ! (P 04/02/01)

\subsubsection{Se démerder et ses dérivés}

De quelqu'un qui se débrouille, on peut dire aussi, de façon plus expressive, qu'il se démerde.

Avant de trouver un salarié français qui n'est pas capable de se démerder, il faut se lever tôt ! (F 24/02/07)

On connait par ailleurs l'expression populaire Article 22 : chacun se démerde comme il peut. Et qui n'a pas entendu les impératifs pseudo-allemands Démerden sie sich et Démerdieren sie sich (orthographiés avec ou sans accent aigu) ? En voici un exemple :

Dans une manifestation publique, on est en droit d'espérer plus de clarté, de lisibilité, de pédagogie. Là, c'est : «Demerden Sie sich ! » Ou presque. (L 20/09/07)

D'après Bernet \& Rézeau $(2008,231)$, « ces formes impératives de pseudoverbes allemands calqués sur le français se démerder offrent un caractère à la fois ludique et euphémique ».

Les dérivés du verbe se démerder incluent le nom démerde (qui figure dans les mêmes tournures que le nom débrouille : on a donc aussi roi de la démerde, école de la démerde, etc.), le nom et l'adjectif démerdard (qui rappellent le nom et l'adjectif débrouillard), le nom démerdeur et l'adjectif démerde. Exemples :

Au passage, elle a appris «la démerde». Indispensable «pour vivre dans l'une des villes les plus chères du monde avec un salaire de serveuse ». (CL 25/09/12)

Cet homme qui parle toujours à voix basse, et dont le téléphone vibre sans discontinuer, est devenu une pièce centrale du dispositif de François Hollande. «Un démerdard », résume l'eurodéputé PS Stéphane Le Foll. (Ex 23/11/11)

La juge lui laisse une chance : s'il trouve un boulot dans les dix jours, il n'ira pas en prison pour un bête problème de scooter. Et c'est parce que Zim est plus 
démerdard que cossard qu'il en décroche un rapidement. (Ex 15/08/05)

Je compte d'abord sur moi avant de compter sur Pôle emploi. Ça va le faire ; je suis un démerdeur... (SO 17/01/12)

Je voulais surtout réussir et j'étais un peu « démerde ». (NR 27/09/11)

Tous ces exemples confirment une fois de plus que la débrouille n'est pas seulement un comportement saillant; elle est également valorisée.

\subsubsection{Système D}

À l'étranger qui ne comprend pas ce qu'est le système D, Geoffroy (2005, 86) apporte une réponse exhaustive ; en disant que c'est à la fois « $\mathrm{D}$ comme débrouillard » et «D comme démerdard », il fait écho au commentaire déjà fort ancien du sous-lieutenant Robert Porchon, mort pour la patrie en 1915 (Porchon 2008, 141 ; Rézeau 2014, 199) :

Pour les Dames mettons que ça veuille dire qu'on se débrouille. Il existe pour les militaires un mot plus énergique et qui appartient au langage des camps.

L'anthropologue américaine Deborah Reed-Danahay, elle, est une « dame» : aucune surprise que, pour elle, la lettre D renvoie au verbe débrouiller (Reed-Danahay 2007, 122). Charles Timoney, en revanche, vit en France depuis le milieu des années quatre-vingts ; il refuse le lien avec le radical débrouill- :

When faced with some conundrum, people say that they will have to resort to système $\mathrm{D}$ to solve it. But what is this « $\mathrm{D}$. » ? There is a verb starting with $\mathrm{D}$, « se débrouiller », that means to find a way round problems and you may innocently believe that this is the $\mathrm{D}$ in question. Not a bit of it. The truth is much more vulgar - « se démerder», which literally means to get yourself out of the shit. This is why you only use the verb's initial. (Timoney 2007, 132)

Les séquences système débrouille et système démerde existent, n'en déplaise à Timoney, mais système $D$ est beaucoup plus courant. Le terme est souvent accompagné d'adjectifs tels que fameux ou célèbre, qui en soulignent la saillance. Ardagh $(1989,503)$ fait allusion au « célèbre système $\boldsymbol{D}$, cet élément essentiel de la vie française » qui « redonne des proportions humaines à la rigidité des procédures officielles » et grâce auquel les Français parviennent à « couper au plus court et à circonvenir certaines absurdités bureaucratiques ». Pour un exemple avec l'adjectiffameux, on peut se tourner vers le passage qui suit, retenu puisqu'il permet d'anticiper sur un slogan dont il sera question plus loin :

Le premier choc pétrolier (1973-1974) entraine une hausse du carburant, et une authentique psychose s'empare des consommateurs. La seconde crise pétrolière de 1979 renforce encore le malaise général. Le fameux système D fait fureur, c'est à qui imaginera les trouvailles les plus ingénieuses, des spots TV affirment : « Nous en France, on n'a pas de pétrole mais on a des idées $\gg .^{10}$

${ }^{10}$ Leroy \& Chollet (2007), cité d'après la fiche accessible sur le site web de la librairie

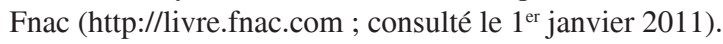


On voit que ce n'est pas seulement pour se soustraire à la myriade de directives, de règlements et de lois qui s'appliquent dans l'une ou l'autre situation concrète, pour échapper au fisc ou pour mettre fin à une situation qui traine depuis trop longtemps parce qu'on a tout voulu faire "selon le règlement », qu'on recourt au système $\mathrm{D}$. Le domaine d'application en est plus vaste, ce qu'illustrent également les exemples d'usage que voici des syntagmes roi / reine du système $D$, qui sont équivalents aux syntagmes roi / reine de la débrouille.

Les étudiants souffrent dans leur grande majorité d'un manque cruel d'argent. Ils sont devenus les rois du système $\mathbf{D}$ grâce notamment aux «plans » friperies où ils peuvent s'habiller et se chausser pour pas cher. (F 18/04/08)

Mon ex-mari m'a toujours dit que j'étais une bonne à rien. C'est faux, je suis la reine du système $\mathrm{D}$. (P 26/03/13)

Observateurs externes et internes s'accordent pour dire que le système $\mathrm{D}$ est un phénomène «national », qu'il s'agisse d'un sport («this well-known national sport », Rogers 1991, 195), d'un passe-temps (« a national pastime», Steele 2006, 101) ou d'une valeur (une «valeur nationale » devenue le «fanion du citoyen Robin des Bois contre le Léviathan administratif », Mény 1992, 16). Les caractéristiques principales du système $\mathrm{D}$ sont le « manque d'orthodoxie » (Lewis 2006, 311) et l'originalité, voire l'imprévisibilité, des démarches adoptées en vue de réaliser l'un ou l'autre objectif. Recourir au système D, c'est tirer le meilleur parti des circonstances, faire preuve d'initiative personnelle, en ne faisant appel qu'aux moyens qu'on a à sa disposition. Roucaute $(2012,166)$ l'a bien résumé :

Le « système D », système « débrouille », ne se déduit pas, ne s'induit pas, ne se décline pas, ne s'apprend pas. Ici, il appelle cette décision, là, pour ce qui semble être un même problème, une autre solution. À cet instant, il proclame la validité d'une procédure, à cet autre, il l'invalide.

\subsubsection{Tuyau}

Les Français prennent plaisir à « se refiler des tuyaux ». Il s'agit de conseils, de renseignements ou d'informations pratiques échangés avec quelqu'un qu'on connait ou avec qui on croit pouvoir s'identifier. Le but est de s'entraider en vue de « fixer » un problème quelconque. Susan Loomis (2001, 77), Américaine établie en France, décrit la pratique comme « an undocumented system which pervades every element of French society ». Elle renchérit en disant (ibid., 79) : « It's part of living here, it's de rigueur, it may be genetic ». On parle de bons tuyaux, séquence relativement courante, et, si le conseil est malencontreux et n'aboutit pas au résultat escompté, de tuyaux crevés. L'idée est en général de faire des économies, d'acquérir des biens à moindre prix ou bien d'obtenir des services. L'achat collectif de vins, par le biais d'une commande partagée dont le 
but est d'obtenir un rabais est une opération parfaitement innocente et profitable à tous. C'est un tuyau tout à fait légal. D'autres tuyaux ne sauraient prétendre à ce statut, puisqu'ils encouragent des pratiques et/ou des démarches qui sont contraires à la loi - par exemple, l'achat chez un grossiste (et donc à des prix réduits) d'articles destinés à un usage privé. Le tuyautage est particulièrement répandu, semble-t-il, dans le domaine financier où il s'est installé afin de réduire l'impact des impôts (directs et indirects), qui en France peuvent être vertigineux.

\subsubsection{Piston, se faire pistonner}

Comme les tuyaux, les pistons jouent un rôle important dans le fonctionnement de divers instruments et machines ; le mot s'utilise en outre en rapport avec le recours à des relations ou des contacts pour s'assurer d'une meilleure chance de succès lors de la recherche d'un poste, d'un logement, d'une place à la crèche ou à l'école de son choix, etc. Le piston est plus répandu dans certains domaines que dans d'autres ; courant dans le privé, il est quasiment tabou dans le secteur public, dans la mesure où l'État est censé se porter garant de l'égalité des citoyens. En outre, il est plus à la portée des « puissants » que des « sous-prolétaires ».

En septembre 2007, l'hebdomadaire Marianne consacra à « la France du piston et des passe-droits » un dossier où se trouve le passage que voici, qui illustre bien de quoi il s'agit :

La scène se passe dans le hall d'un cabinet d'avocat parisien. Comme tous les premiers lundis du mois, les nouveaux stagiaires font connaissance autour de la machine à café. Après avoir décliné leur nom, prénom, âge et cursus universitaire, la question fuse : «Et toi, t'es rentré par qui ? » Une interrogation qui n'a rien d'agressif : chacun des apprentis juristes se plie volontiers à l'exercice et annonce, sans la moindre gêne, que son CV a atterri sur le bureau de la responsable du personnel grâce à un père qui « connait bien le patron », une cousine qui fréquente «le même club de gym qu'une avocate », un frère qui « est sorti avec une secrétaire » ou une copine qui «bosse comme serveuse au restaurant d'en face »! À les écouter, on comprend que la situation n'est pas spécifique à ce cabinet : aux yeux de ces étudiants en droit, espérer trouver un emploi sans avoir de « connexion » relève au mieux de l'idéalisme, au pis de la naïveté. Et, à moins de 25 ans, certains sont déjà passés maitres dans l'art de «se faire pote ». (Ma 22/09/07)

À la différence du mot tuyau, le mot piston s'utilise communément avec l'article partitif :

Les jeunes issus de l'université sont souvent réticents à l'idée de faire jouer les relations, qu'ils assimilent immédiatement à du piston. (Ex 25/09/03)

Grâce à du « piston », la jeune recrue se retrouve affectée à Saint-Maixent-l’École, dans la musique. (SO 08/12/10)

De quelqu'un qui a obtenu une faveur en faisant appel à des contacts qu'il entretient avec des personnes bien placées, on dit qu'il a du piston ou qu'il s'est 
fait pistonner :

Combien de fois lui a-t-on demandé si elle s'était fait pistonner par son père ? (P 10/07/07)

Ce Rabelaisien aux racines alsaciennes a fait son service militaire chez les chasseurs alpins. « La seule fois où je me suis fait pistonner ». (BR 13/12/10)

Il y a du piston en médecine veut dire que, dans le domaine professionnel de la médecine, le phénomène s'observe avec une certaine régularité.

\subsection{EXPRESSIONS IMAGÉES}

Trois expressions imagées peuvent être mises en rapport avec la débrouille. Ce sont tirer (ou sortir) son épingle du jeu, faire flèche de tout bois et faire feu de tout bois. Toutes trois ont le plus souvent une connotation positive et renforcent dès lors l'idée que la débrouille est un comportement valorisé.

\subsubsection{Tirer / sortir son épingle du jeu}

À l'origine de l'expression imagée tirer (ou sortir) son épingle du jeu se trouve un jeu d'adresse pour petites filles dont les origines remontent au 15 siècle (Duneton 2001, 218 ; Guillemard 2007, 379 ; Guilleron 2008, 78). Parvenir à tirer son épingle du jeu, c'est se débrouiller en faisant preuve d'adresse et de dextérité.

Certains parviennent toutefois à tirer leur épingle du jeu. Jérôme, 23 ans, sansabri depuis plusieurs mois, a trouvé un studio. (OF 31/12/08)

On reste un peu sur notre faim, mais bon... On tire malgré tout notre épingle du jeu. $(\operatorname{Pr} 04 / 08 / 10)$

\subsubsection{Faire flèche de tout bois}

L'expression faire flèche de tout bois date du 17e siècle (Guilleron 2010, 105). Du point de vue de l'archer, mieux valait se débrouiller en se servant de flèches faites d'un bois médiocre que de n'avoir pas de flèches du tout. Même les flèches de mauvaise qualité peuvent atteindre leur cible et contribuer à la réalisation du but. Exemples d'usage :

En 1793, les armées de la République manquent de tout. Et l'on fait flèche de tout bois. (Pt 26/04/07)

Trois candidats de droite font flèche de tout bois pour l'abattre. (F 16/02/08)

\subsubsection{Faire feu de tout bois}

$\mathrm{Au}$ lieu de faire flèche de tout bois, on peut faire feu de tout bois. Le but est le même : mettre tout en œuvre afin de réussir, réaliser ses objectifs par tous les moyens possibles. Le résultat lui aussi est pareil : on atteint son but sans attacher trop d'importance au(x) moyen(s) utilisé(s). Faire feu de tout bois s'est répandu dans l'usage au cours du $19^{\mathrm{e}}$ siècle. 
$\mathrm{Du}$ clip publicitaire au documentaire en passant par le film d'entreprise, pédagogique, institutionnel... les deux amis feront feu de tout bois. (ML 26/05/07) Pour contrer les critiques et sa baisse dans les sondages, le chef de l'État fait feu de tout bois. Au risque parfois de provoquer quelques imbroglios. (Pt 21/02/08)

\subsection{SLOGANS ET DICTONS}

En France, on n'a pas de pétrole, mais on a des idées est un slogan dont la souplesse n'a pas fini d'étonner. Impossible n'est pas français et Ce n'est pas la mer à boire, quant à eux, sont des dictons ou des dit-on ${ }^{11}$ qui ont parlé à l'imagination des inventeurs de slogans. Les deux premiers vantent l'inventivité des Français et leur détermination à se débrouiller en cas de difficulté ; le troisième souligne l'idée que la tâche, quelle qu'elle soit, peut être accomplie.

\subsubsection{En France, on n'a pas de pétrole, mais on a des idées}

«Le slogan est archiconnu, et inusable : en France, on n'a pas de pétrole, mais on a des idées » $(\operatorname{Pr} 26 / 10 / 10)$. Pour en retracer les origines, il faut remonter à la campagne menée par l'État français dans la foulée de la crise pétrolière qui, aux années soixante-dix, s'était abattue sur l'Europe tout entière. Le gouvernement avait créé en 1974 une agence (l'AEE, ou Agence pour les économies d'énergie), dont l'une des premières initiatives fut la production, en 1976, d'un spot télévisé (cf. § 3.1.5) mettant en scène un pêcheur qui tenait les propos que voici :

En France, on a toutes sortes de choses. On a la meilleure cuisine du monde, une industrie puissante, la pétanque, l'histoire glorieuse. On a aussi une situation géographique privilégiée, la tour Eiffel... et la pêche à la ligne. Oui, en France, on a tout ça. Et bien plus encore. Pourtant, une chose nous manque, une chose essentielle : le pétrole. Le pétrole, nous sommes obligés de l'acheter à d'autres. Cher, trop cher. C'est notre richesse qui s'en va, et notre façon de vivre qui est menacée. Alors, que peut-on faire ? Eh bien d'abord, mieux utiliser l'énergie. Et ça, justement, nous pouvons le faire sans changer notre façon de vivre. À partir du 15 septembre, l'Agence pour les économies d'énergie vous dira comment. Vous verrez... En France, on n'a pas de pétrole, mais on a des idées.

Des versions écourtées du spot original, de nouveaux messages publicitaires, et enfin des chansons très engagées ${ }^{12}$ où le slogan de l'AEE se retrouve en filigrane, en ont assuré la postérité. En octobre 2008, plus de trente ans après sa création, il occupa la onzième place dans un classement établi à la suite d'un sondage lors duquel on avait proposé aux personnes interrogées

11 Termes utilisés ici pour faire ressortir ce qu'ont en commun ces deux tournures dont seule la première peut prétendre au statut de sagesse populaire, ce qui en ferait un proverbe.

12 Par exemple J'suis français de Marc Charlan (1976) et Ils ont le pétrole, mais c'est tout de Michel Sardou (1979) - et plus récemment On n'a pas de pétrole du rappeur Rockin Squat, alias Mathias Crochon (2010). 
une vingtaine de slogans issus de campagnes gouvernementales menées depuis les années soixante-dix. Le sondage lui-même avait été organisé dans la foulée de la publication d'un ouvrage (Benoît \& Scale 2008) qui faisait le tour d'une cinquantaine de campagnes gouvernementales s'appuyant sur une variété d'images, de slogans, de spots, etc. et où est souligné (op.cit., 9) un fait « significatif», à savoir que « la connaissance de ces slogans se confirme même chez des jeunes qui, au moment de la première diffusion de la campagne, n'étaient pas encore nés ». De nombreuses occurrences plus ou moins littérales du slogan de l'AEE, postérieures au sondage, attestent par ailleurs de sa popularité, non seulement quand la thématique abordée est celle du pétrole, des économies d'énergie ou des énergies nouvelles :

L'heure d'été a été instaurée en 1976, trois ans après le choc pétrolier. En France, à l'époque, on n'avait pas de pétrole mais des idées... (ER 25/10/08)

Si tout va bien, on verra rouler d'ici quelques mois les premières voitures alimentées en essence bactérienne. En France, on n'a peut-être pas de pétrole, mais on a, effectivement, des idées. (Ex 14/01/10)

C'est bien connu, en France, on n'a pas de pétrole, mais on a des idées, ou du moins du bon vin, surtout dans le Bordelais... (SO 10/07/14)

...mais aussi dans d'autres contextes qui documentent la débrouille des Français :

C'est bien connu, en France on n'a pas de pétrole mais on a des idées. La municipalité de Palmas, avec à sa tête Paul Redon, en a apporté la preuve pour mener à bien son projet de construction d'un nouveau groupe scolaire. (ML 19/10/09)

La France est un pays formidable. Nous n'avons toujours pas de pétrole, mais nous avons encore des idées. Aujourd'hui, alors qu'il faut résoudre la question des retraites, chacun planche pour trouver des solutions originales. (JF 12/06/10) Comme on n'a pas de pétrole, il a fallu avoir des idées. Nous avons concentré notre masse salariale sur neuf pros. Est-ce que ça va payer ? Ça, on ne le saura qu'après. (SO 26/09/14)

\subsubsection{Impossible n'est pas français}

«Impossible n'est pas français / Ce proverbe, on le connait », chantait aux années soixante Sheila (née Annie Chancel), chanteuse pop de nationalité française. Il s'agit, enchainait-elle, d' « une phrase que j'aime bien ». Une phrase que d'autres ont décrite à l'aide de termes tels que maxime et dicton et qui indique qu'il y a toujours une solution, quel que soit le problème :

Dans le milieu des jeunes enseignants, il est une maxime qu'on apprend dès la première rentrée : «Impossible n'est pas français ». (F 25/05/00)

Elle répète pour s'encourager ses trois dictons préférés : «Qui ne tente rien, n'a rien. Qui va doucement, va surement. Impossible n'est pas français ! (ML 26/05/07)

Dans cette «phrase célèbre dans la mémoire collective des Français » (Henry 2006, 109), qu' on fait le plus souvent remonter à Napoléon $1^{\text {er }}$, certains 
voient une « composante du tempérament français » (ibid.), « un principe sacré ancré dans la culture française » (Roucaute 2012, 166). Ces quelques mots, qu'ils soient proverbe, maxime ou dicton (ou autre chose encore), continuent à jouir d'une belle popularité dans l'usage contemporain de la langue :

Impossible n'est pas français. Même de vendre des programmes TV français aux Asiatiques, y compris des documentaires! (T 19/03/02)

Sacré programme pour un jeune proviseur. Mais, à voir son désir de faire avancer le collège, on a envie de dire : « Impossible n'est pas français. » (SO 01/09/08)

En principe, le nombre de fois que le dicton Impossible n'est pas français devrait pouvoir être utilisé est légion, car, ainsi que l'ont constaté Corbett (2007, 249-250) et Bard (2010, 314), des routines telles que Ce n'est pas possible, C'est pas possible, Pas possible et C'est impossible, prononcées d'un ton sec, sont monnaie courante dès que, pour l'une ou l'autre raison, un service sollicité ne peut être rendu, ou semble ne pas pouvoir l'être. Que faire pour obtenir satisfaction? On peut se mettre à râler (Peeters 2013b) ou faire preuve de débrouille. Mieux vaut ne pas parer un Pas possible avec un Impossible n'est pas français, qui ne ferait qu' aggraver la situation (Timoney 2007, 85). Le risque de se faire insulter, de s'entendre dire qu'on « se fout » de l'interlocuteur ou qu'on « se fout de sa gueule » est des plus réels. En effet, se trouvant dans l'impossibilité de contredire un dicton que tous les Français reconnaissent et apprécient s'il est utilisé au moment propice -, l'interlocuteur n'a d'autre recours que de s'en prendre à l'intégrité morale de son adversaire. Malone $(2008,49)$ offre à son lecteur la morale que voici :

«Impossible n'est pas français ! » It simply isn't the French way to accept defeat in the face of adversity. However, if a French person tells a non-French person that something is impossible, then it is impossible.

$\mathrm{Du}$ dicton au slogan, il n'y a parfois qu'un pas. Ce pas a notamment été franchi en 2011, et de nouveau en 2014. En 2011, Impossible n'est pas français est devenu le slogan du Crédit agricole. La banque a parrainé une série d'initiatives et de projets culturels ou économiques ambitieux, innovants et souvent étonnants, lancés par des associations ou des entreprises « au grand cœur » en vue de résoudre l'un ou l'autre problème humanitaire (par exemple, comment rapprocher les œuvres d'art du grand public, comment faciliter la distribution du lait frais dans les petites communautés, que faire de l'excès de poissons pêchées à la criée, etc.). Elle a ensuite financé une série de minimétrages diffusés sur TF1 du 4 avril au 30 juillet, dans lesquels un journaliste anglais se rend aux quatre coins de la France, à la découverte de ces initiatives et de ces projets. Trente-neuf émissions intitulées Impossible n'est pas français ont été produites, une par initiative parrainée, avec mention du Crédit agricole au début et à la fin de chaque segment. En 2014, c'est l'équipe nationale de football (« les Bleus ») qui s'est approprié le slogan pour la Coupe du monde 
au Brésil. Le choix de slogan avait été basé, comme d'habitude, sur un vote en ligne, procédure suivie par chacune des équipes qualifiées. Pour la France, le choix était entre En route vers l'exploit, Impossible n'est pas français ou Un pour tous, tous pour la France. La victoire ne lui a pas pour autant été réservée : les Bleus ont été éliminés dans les quarts de finale par les Allemands.

\subsubsection{Ce n'est pas la mer à boire}

Louise, protagoniste du roman To Algeria, with love (Ruta 2011), se souvient avec affection des nombreuses leçons reçues de Wally, travailleur algérien qui allait devenir le père de son premier fils. L'une de ces leçons concernait l'apprentissage de la langue française, cruciale pour cette étudiante newyorkaise partie étudier en France :

Learning French, he insisted, was not that big a deal. «Ce n'est pas la mer à boire. » It's not like swallowing the sea. He had a great supply of odd phrases they never taught us at school. (Ruta 2011,35)

Drôle d'expression, certes - du point de vue de quelqu'un qui n'a pas l'habitude de s'exprimer en français, où elle est pourtant tout à fait courante, bien plus courante que la version affirmative qui, elle, est plus ancienne mais a pratiquement disparu :

L'État doit maintenant débloquer sa part. Quarante-huit millions sur cinq ans, ça fait 10 millions en moyenne par an, ce n'est pas la mer à boire ! (P 16/06/09) Le bac ne vaut plus rien, c'est juste une clé, ce n'est pas valorisant, ce n'est pas la mer à boire, il faut de bonnes notes toute l'année et on s'en sort. (SO 18/06/10)

Protéger l'océan, ce n'est pas la mer à boire! était en 2011 le slogan de l'association Attention Mer Fragile. La campagne menée sous ce slogan avait pour objectif de promouvoir le tourisme maritime responsable. La protection du littoral est un autre objectif de cette association bretonne fondée en 2003, dont le programme ne concerne évidemment pas que la Bretagne. L'association organise des journées Mer Fragile, destinées à sensibiliser le grand public, à commencer par les enfants, à la nécessité de sauver nos océans des nombreux dangers qui les menacent. Il faut pour cela faire preuve (d'un minimum) de débrouillardise.

\section{CONCLUSION}

On dit parfois que l'image du Français débrouillard a fait son temps et ne se manifeste plus avec la même ténacité qu'autrefois. Les témoignages et les faits de société et de langue présentés dans cette étude montrent qu'il n'en est rien. A-t-on affaire à un défaut ou à une qualité ? Dans l'ensemble, les données réunies plaident en faveur de la débrouille (ou de l'art de la débrouille), qui revêt ainsi le statut de valeur culturelle française. Dans la vie, il faut savoir se débrouiller. Certes, c'est une qualité qui se prête à des abus ; mais connait-on une qualité qui ne s'y prête pas? 


\section{BIBLIOGRAPHIE}

Ardagh, J. (1989), Ces drôles de Français. Voyage au cœur de la France profonde, Paris : Pierre Belfond (traduit de l'anglais par Jean-Paul Mourlon).

Asselin, G. et Mastron, R. (2005), Français - Américains. Ces différences qui nous rapprochent, Paris : Alban Éditions (traduit de l'anglais par Marianne SeïteKarner).

Bard, E. (2010), Lunch in Paris, Sydney : Harper Collins.

Baxter, J. (2006), We'll Always Have Paris. Sex and Love in the City of Light, London : Bantam Books.

Benoît, J.M. et Scale, J. (éd.) (2008), Bleu, blanc, pub. Trente ans de communication gouvernementale en France, Paris : Cherche-Midi.

Bernet, C. et Rézeau, P. (2008), On va le dire comme ça. Dictionnaire des expressions quotidiennes, Paris : Balland.

Bourrier, M. (1999), Le nucléaire à l'épreuve de l'organisation, Paris : PUF.

Corbett, B. (2007), A Town like Paris, Sydney : Hachette Australia.

d'Iribarne, P. (2006), L'étrangeté française, Paris : Seuil.

Duneton, C. (2001), La puce à l'oreille. Les expressions imagées et leur histoire, Paris : Balland.

Goddard, C. (2011). Semantic Analysis. A Practical Introduction, Oxford : Oxford University Press.

Goddard, C. et Wierzbicka, A (ed.) (2002), Meaning and Universal Grammar. Theory and Empirical Findings, Amsterdam : John Benjamins.

Greene, J. (2002). French Spirits. A House, a Village, and a Love Affair in Burgundy, Sydney : HarperCollins.

Guillemard, C. (2007), Secrets des expressions françaises, Paris : Bartillat.

Guilleron, G. (2008). À la queue leu leu. Origines d'une ribambelle d'expressions populaires, Paris : First.

Henry, G. (2006). L'habit ne fait pas le moine. Petite histoire des expressions, Paris : Tallandier / Points.

Kerbrat-Orecchioni, C. (2008). «Les interactions en site commercial. Des interactions “polies" ». In C. Kerbrat-Orecchioni et V. Traverso (éd.), Les interactions en site commercial. Invariants et variations, Lyon : ENS, 105-137.

Klein, J. (2013), Reputation Economics. Why who you know is worth more than what you have. New York : Palgrave Macmillan.

Leroy, A. et Chollet, L. (2007), L'album de ma jeunesse 70-80. Mon enfance, mon adolescence, Paris : Presses de la Cité. 
Lewis, E. (2006), Left Bank Waltz. The Australian Bookshop in Paris, Sydney : Random House.

Loomis, S. (2001), On Rue Tatin, Sydney : HarperCollins.

Malone, M. (2008), Ooh La La ! How to be Infuriatingly French, Chichester : Summersdale.

McGregor, F. (1993), Au Pair, Melbourne : McPhee Gribble.

Mény, Y. (1992), La corruption de la République, Paris : Fayard.

Neuwirth, R. (2011), Stealth of Nations. The Global Rise of the Informal Economy, New York : Pantheon.

Peeters, B. (2006a), « She'll be right vs On va s'arranger. Étude ethnophraséologique », RSP 19/20: 71-89.

----- (ed.) (2006b), Semantic Primes and Universal Grammar. Empirical Evidence from the Romance Languages, Amsterdam : John Benjamins.

-----. (2010), « La métalangue sémantique naturelle. Acquis et défis », in J. François (éd.), Grandes voies et chemins de traverse de la sémantique cognitive, Leuven : Peeters, 75-101.

-----. (2012), « L'interculturel servi à la sauce MSN, ou À quoi sert la métalangue sémantique naturelle? ». In N. Auger, C. Béal \& F. Demougin (éd.), Interactions et interculturalité. Variété des corpus et des approches, Bern : Peter Lang, 149-180.

-----. (2013a), « Language and cultural values : towards an applied ethnolinguistics for the foreign language classroom ». In B. Peeters, C. Béal et K. Mullan (ed.), CrossCulturally Speaking, Speaking Cross-Culturally, Cambridge : Cambridge Scholars Publishing, 231-259.

-----. (2013b), « Râler, râleur, râlite. Discours, langue et valeurs culturelles », in C. Claudel, P. von Münchow, M. Pordeus, F. Pugnière-Saavedra et G. Tréguer-Felten (éd.), Cultures, discours, langues. Nouveaux abordages, Limoges : LambertLucas, 117-141.

-----. (2014), « On va s'arranger / On s'arrangera : étude ethnophraséologique de deux actes (généralement) rassurants ». Scolia 28 : 129-149.

Porchon, R. (2008), Carnet de route, suivi de lettres de Maurice Genevoix et autres documents, T. Joie (éd.). Paris : Table Ronde.

Rézeau, P. (2014), « Aspects de la phraséologie du français de France à travers des correspondances de combattants de 1914-1918», in A. Farina et V. Zotti (éd.), La variation lexicale des français. Dictionnaires, bases de données, corpus, Paris : Honoré Champion, 189-213.

Rogers, S.C. (1991), Shaping Modern Times in Rural France. The Transformation and Reproduction of an Aveyronnais Community, Princeton : Princeton University Press. 
Roucaute, Y. (2012), Éloge du mode de vie à la française. The French way of life, Monaco : Éditions du Rocher.

Ruta, S. (2011), To Algeria, with Love, London : Virago.

Steele, R. (2006), The French Way. The Keys to the Behavior, Attitudes, and Customs of the French, New York : McGraw-Hill.

Timoney, C. (2007), Pardon my French. Unleash your Inner Gaul, London : Penguin.

Wierzbicka, A. (2006a), « Les universaux empiriques du langage. Tremplin pour l'étude d'autres universaux humains et outil dans l'exploration de différences transculturelles », Linx $54:$ 151-179.

(2006b), « Sens et grammaire universelle. Théorie et constats empiriques », Linx 54 : 181-207 (une version abrégée et révisée de ce texte a été publiée en 2006 dans les Cahiers Ferdinand de Saussure 59 : 151-172). 